\title{
Consistent Behavioral Abstractions of Experimental Frame
}

\author{
Sangeeth saagar Ponnusamy ${ }^{*}$ \\ Airbus Operations SAS, 316 Route de Bayonne, 31060, Toulouse, France \\ Vincent Albert ${ }^{\dagger}$ \\ CNRS, LAAS, 7 Avenue du Colonel Roche, 31400, Toulouse, France \\ Patrice Thebault ${ }^{\ddagger}$ \\ Airbus Operations SAS, 316 Route de Bayonne, 31060, Toulouse, France
}

\begin{abstract}
A general notion of the simulation fidelity in system verification and validation is explained in the modeling $\&$ simulation framework of the experimental frame and discussed in the context of modeling abstractions. A formal method for fidelity measurement is presented for semantic compatibility through abstraction inclusion relations. Behavioral modeling abstractions of dynamical systems consistent with simulation objectives are explained in the experimental frame formalism using bisimulation relations and their approximations. Property-preserving bisimulation relations consistent with the simulation objectives are explained in the context of hierarchical abstractions and composition of experimental frame components. The behavioral fidelity problem is presented by finding bisimilarity preserving surjection maps of desired abstraction precision. In abstraction of linear time invariant systems, the paper discusses the problem of extending the classical results to finding surjections whose null space is a controlled invariant subspace with respect to fidelity requirements. Applicability and derivability of experimental frames are discussed through such consistent abstractions. In conclusion, challenges in research and implementation of a semi-formal unified fidelity framework encompassing both syntactic and semantic aspects are discussed.
\end{abstract}

\section{Nomenclature}

$\begin{array}{ll}\mathrm{EF} & =\text { Experimental Frame } \\ \mathrm{H} & =\text { linear surjection map } \\ \mathrm{M} & =\text { model } \\ \mathrm{X} & =\text { inputs to model } \\ \mathrm{Y} & =\text { outputs of model } \\ \mathrm{S} & =\text { states of model } \\ \alpha & =\text { abstraction } \\ \beta & =\text { derivability } \\ \gamma & =\text { applicability } \\ f_{\mathrm{B}} & =\text { bisimulation function } \\ \varepsilon & =\text { abstraction precision } \\ \varphi & =\text { requirement } \\ \prod & =\text { transition system }\end{array}$

\section{Introduction}

Modelling \& Simulation (M\&S) is being widely used as a means to conceive, develop and test complex engineering systems. In this Model Based context of systems engineering, Verification and Validation (V\&V)

\footnotetext{
* Doctoral Researcher, Simulation Architecture \& R\&T, 316 Route de Bayonne, 31060, Toulouse.

${ }^{\dagger}$ Assisstant Professor, Systems Engineering \& Integration, 7 Avenue du Colonel Roche, 31400, Toulouse.

* Systems Simulation Modeling Expert, Simulation Architecture \& R\&T, 316 Route de Bayonne, 31060, Toulouse.
} 
determine the compliance of simulation products with their specifications and fitness for their intended use respectively. However in system validation through simulation, a 'distance to reality' requirement is seldom expressed even if the context of use is well known. The effectiveness of simulation in reproducing the reality i.e. realism of simulation, motivates the question of how to quantify the distance between a system and its simulation behavior with respect to its $\mathrm{V} \& \mathrm{~V}$ objectives.

A general overview of this fidelity problem and its formal assessment based on the structural and behavioral aspects is given in Ref.12. In this paper, behavioral abstractions of system through approximate bisimulation relations ${ }^{10}$ are explained in the established M\&S concept of experimental frames ${ }^{9}$. The paper is structured as follows, the concepts of fidelity and abstractions are briefly explained followed by proposition on the formal approach in abstraction quantification with respect to the simulation objectives.

\section{Abstractions in M\&S}

A model is always an abstraction of the reality it is intended to represent and abstraction is essentially a complexity reduction operation, for which, there exist myriad classes and types ${ }^{15}$. According to IEEE 610.12-1990 standard, a model is formally defined as an approximation, representation, or idealization of selected aspects of the structure, behavior, operation, or other characteristics of a real-world process, concept, or system.

In modeling and simulation of complex systems, the difficulty is often finding and implementing consistent and valid abstractions to model the simulated system with respect to simulation requirements. This is more so true in developing a complex simulation product where the component models can be developed by different stakeholders and a common frame of reference must exist in terms of choosing, building and implementing abstractions consistent with the objectives. Extensive developments are being made in formal verification of models. However, owing to the fact that most of the models are found to be verified but invalid, the focus must be on developing models consistent with objectives apriori rather than verifying it post priori. This challenging top down approach of building models with consistent abstraction will help increase confidence in simulation results in addition to improving the reusability which in turn reduces cost and time of system development ${ }^{12,11}$.

\section{Simulation Fidelity}

In system validation through simulation, the environment of this System Under Test (SUT) is simulated with the help of models which in turn could be physical or virtual or a combination of both. In modeling these environmental systems, abstractions introduce a 'distance' to the reality and this distance must be 'quantified' in order to meaningfully assess the SUT according to the V\&V objectives.

In this paper, Fidelity is defined as this notion of 'distance to reality' similar to the definitions ${ }^{16}$ of Implementation Study Group (ISG) of Simulation Interoperability Standards Organization (SISO). In Ref.12, the problem of finding the right level of modeling abstractions in satisfying the fidelity requirements and a formal proposition for its quantification was proposed in terms of structural and behavioral compatibility criteria. In this top down process of simulation product development a relative measure called the relative fidelity or simulation model fidelity is introduced instead of an absolute fidelity measure which is neither feasible nor useful. Denoting this quantification by a parameter, $\varepsilon$, this relative fidelity is defined as a measure of closeness of abstraction between the simulation user requirements called Simulation Objective of Use (SOU) and model capabilities called Simulation Domain of Use (SDU). It is expressed as,

$$
\mathrm{M}_{\mathrm{F}}^{\mathrm{sim}}=\varepsilon_{\mathrm{SDU}} / \varepsilon_{\mathrm{SOU}}
$$

The perspectives of SDU and SOU can be formalized in terms of experimentation context of the SUT. Such a common frame for implementing abstractions was given by the concept of experimental frame from the theory of $\mathrm{M} \& \mathrm{~S}$.

\section{A. Experimental Frame}

In the context of studying a system through simulation, the concept of experimental frame (EF) defines controllability and observability means to stimulate and observe the SUT temporal evolution under the experimentation conditions ${ }^{9}$. An experimental frame is composed of stimulant (Generator), observer (Transducer) and assessor (Acceptor) components in addition to the components simulating the environment (EM). In general, it is written as composition $(\Sigma)$ of different models, $\mathrm{M}_{\mathrm{i}}$ where $\mathrm{i}$ is the number of models. 


$$
\mathrm{EF}_{\text {sim }}=\sum_{\mathrm{i}=1 . . \mathrm{n}} \mathrm{M}_{\mathrm{i}}
$$

The components models in Eq.(2) are given as a tuple with three elements of time (T), Input $\mathrm{X}$ and output $\mathrm{Y}$ as follows

$$
\mathrm{M}_{\mathrm{i}}=\left\langle\mathrm{T}, \mathrm{X}_{\mathrm{n}_{\mathrm{X}}}^{\mathrm{i}}, \mathrm{Y}_{\mathrm{n}_{\mathrm{Y}}}^{\mathrm{i}}>\right.
$$

where $n_{X}, n_{Y}$ define the number of input and outputs respectively. Different such models can be interconnected and hierarchically composed to form an EF to perform test on SUT. For a description of classification of components and EF validity assessment through trace inclusion criteria with an example, see Ref.11.

In general, an EF is built to assess the SUT according to the given V\&V objectives. In this context, a system validity assessment is viable by simulation only when the simulation vis à vis EF itself is valid. The system validity assessment by simulation thus becomes validation against the requirements $\varphi$ is given by

$$
\varphi_{\mathrm{i}=1 \ldots \mathrm{n}}=\varphi_{\text {sys }} U \varphi_{\text {sim }}
$$

where $\varphi_{\text {sys }}$ and $\varphi_{\text {sim }}$ be system and simulation requirements respectively on the system and its representation i.e. model.

The objectives of the experiment could be temporal, functional or architectural. In essence, an EF is sequentially derived from high level definition called Intention Of Use (IOU) such as use cases given by the simulation user. The refinement of $\mathrm{V} \& \mathrm{~V}$ plan described through use cases to define $\mathrm{EF}$ architecture and behavior hierarchically is the subject of another study and is not discussed in this paper. It is assumed that the use case definitions are valid i.e. requirements are validated and its subsequent $\mathrm{EF}$ architecture is consistent with objectives. The problem then becomes how to ensure the EF behavior consistent with user requirements or more specifically, how to abstract the EF component model behavior such that the resulting composition as an EF will result in a behavior at the EF-SUT interface consistent with requirements.

\section{Experimental Frame Fidelity}

The behavioral fidelity problem can be posed as the compatibility of EF behavior at the interface to SUT from the perspectives of SDU and SOU. In other words, relative fidelity is the distance between the output of an expected $\mathrm{EF}$ i.e. $\mathrm{EF}_{\mathrm{SOU}}$ and available $\mathrm{EF}$ i.e. $\mathrm{EF}_{\mathrm{SDU}}$. Through inclusion relation between two such frames whose outputs are denoted by $\mathrm{Y}_{\mathrm{EF}}^{\mathrm{SOU}}$ and $\mathrm{Y}_{\mathrm{EF}}^{\mathrm{SDU}}$ respectively, the fidelity of an experimental frame is said to be correct if

$$
\mathrm{Y}_{\mathrm{EF}}^{\mathrm{SOU}} \sqsubseteq \mathrm{Y}_{\mathrm{EF}}^{\mathrm{SDU}}
$$

This general inclusion is applicable for both syntactic and semantic compatibility ${ }^{12}$. This paper deals with the behavioral compatibility criteria and the aspects of architectural compatibility are not discussed. The scope is limited to complexity reduction in the class of dynamic systems, namely, semantic compatibility. The following figure represents this notion, the behavior of $\mathrm{EF}$ i.e. output $\mathrm{Y}_{\mathrm{EF}}$ from $\mathrm{EF}$ composed on different components such as Generator, Transducer, Acceptor and environment model $\left(\mathrm{EM}_{s 1 . . n}\right)$ is illustrated. In addition, SUT output fed to EF can influence EF output fed to SUT in case of a feedback.

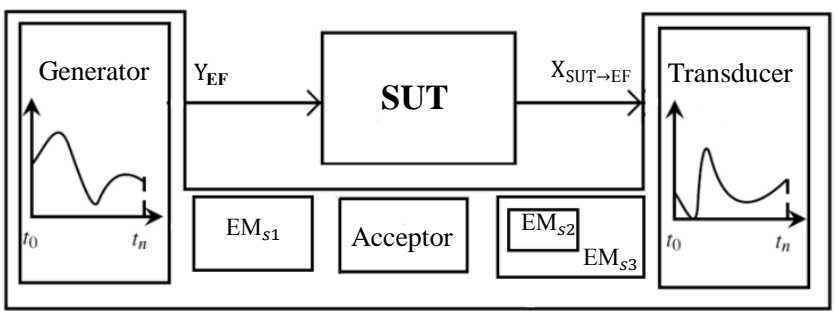

Figure 1: Experimental Frame Fidelity

American Institute of Aeronautics and Astronautics 
The problem then becomes how to abstract the EF components such that the distance or 'error' introduced by the abstraction operation results in EF behavior consistent with user requirements. This abstraction operation over state space of size $\mathrm{n}_{\mathrm{s}_{\mathrm{i}}}^{\mathrm{j}}$ is defined as follows,

Definition: Let the abstraction, $\alpha_{i}^{j}: \mathrm{R}^{\mathrm{n}_{\mathrm{s}_{i}}^{\mathrm{j}} \rightarrow \mathrm{R}^{\mathrm{n}_{\mathrm{i}}} \text {, }}$, be a surjection, mapping a model $\mathrm{M}_{\mathrm{i}}^{\mathrm{j}}$ to its abstract version, $\mathrm{M}_{\mathrm{i}}^{\mathrm{j}+1}$, where $n_{s_{i}}^{j}>n_{s_{i}}^{j+1}$. The hierarchy of abstractions are related by a binary relation forming a partial order. The height of the lattice, $\mathcal{L}_{\alpha_{\mathrm{i}}}$ is given by the size of $\mathrm{j}=\{1 . . \mathrm{n}\}$ and the position at the lattice corresponds to abstraction level.

The valid set of abstractions among the different set of abstractions for a given model $\mathrm{i}=\mathrm{n}$ is defined by

$$
\forall \mathrm{a} \in \mathrm{j}, \exists\left\{\alpha_{\mathrm{i}}^{\mathrm{j}}\right\} \vDash\left\{\varphi_{1}, \varphi_{2} \ldots \varphi_{\mathrm{z}}\right\}
$$

where $\varphi_{\mathrm{i}=1 . . \mathrm{z}}$ are the requirements defined in formalism such as temporal logic as in Eq.(4).

The EF fidelity problem as defined in Ref.12, then can be represented as problem of finding abstractions such that the model behaviour is bounded by the required fidelity distance, $\varepsilon_{\mathrm{f}}$, with respect to the expected behaviour at the EF interface to SUT.

$$
\alpha_{\mathrm{i}}^{\mathrm{j}} \mid \mathrm{Y} \mathrm{EF}_{\varepsilon_{\mathrm{f}}}^{\mathrm{SDU}} \mathrm{Y}_{\mathrm{EF}}^{\mathrm{SOU}}
$$

The validity assessment of abstractions is evaluated through trace inclusion relations ${ }^{11,17}$. However, the difference in our approach is that we intend to relate this abstraction with respect to requirements and quantify it rather than proposing assessment criteria. An abstraction method thus needs to quantify this 'distance' to required level of 'reality'. The following section proposes a method which quantifies the abstraction with bounds on the behavior, followed by integrating the requirements in such an abstraction mapping for behavioral compatibility.

\section{A. Behavioral Abstractions by Approximate Bisimulation Relations}

Bisimulation originally introduced in computer science has been brought to control ${ }^{6}$ and it is a notion that unifies state space equivalence and reduction ${ }^{10}$. Bisimulation could be used for building abstractions resulting in abstract semantics compatible with the simulation objectives in what is termed as Semantic compatibility. Approximate Bisimulation relations developed by Pappas, Girard et al in Ref.10 within the framework of metric transition systems extends this notion of bisimulation to continuous systems.

A metric transition system is a transition system whose outputs are equipped with a metric such as the Euclidean distance. Consider two transition systems $\prod_{1,2}$ which essentially refer to a concrete EF model and its abstraction defined in Eq.(6) as,

$$
\prod_{\mathrm{n}}=<\mathrm{S}_{\mathrm{n}}, \mathrm{X}_{\mathrm{n}}, \mathrm{T}_{\mathrm{n}}, \mathrm{S}_{\mathrm{n}}^{0}, \mathrm{Y}_{\mathrm{n}}, \mathrm{O}_{\mathrm{n}}>, \mathrm{n}=1,2
$$

where

$\mathrm{S}_{\mathrm{n}}$ are the set of states

$\mathrm{X}_{\mathrm{n}}$ are the set of inputs

$T_{n}$ are the transition maps, $T_{n}: S_{n} \times X_{n} \rightarrow 2^{S_{n}}$

$\mathrm{S}_{\mathrm{n}}^{0}$ are the set of initial states $\mathrm{S}_{\mathrm{n}}^{0} \subseteq \mathrm{S}_{\mathrm{n}}$

$\mathrm{O}_{\mathrm{n}}$ are the output maps $\mathrm{O}_{\mathrm{n}}: \mathrm{S}_{\mathrm{n}} \rightarrow \mathrm{Y}_{\mathrm{n}}$ equipped with a metric d.

Approximate Bisimulaion $(\mathrm{AB})$ relations are intended to capture the most significant characteristics of a system dynamics and neglect the less important ones ${ }^{3}$. The degree of approximation is given by the precision of the $A B$ function $(\varepsilon)$ and this precision provides a bound of the distance between the output trajectories of a system and its abstraction. The set of output trajectories, $\{(\mathrm{Y}, \mathrm{X}) \mid \mathrm{Y}=\mathrm{O}(\mathrm{S})\}$, denoted by $\mathcal{L}(\mathrm{T})$ is called the language of the transition system, $\Pi$. The behavioral equivalence between two systems described by homomorphism relation is given here in terms of the observational equivalence i.e. language inclusion and equivalence.

From Ref.10, two metric transition systems $\prod_{1}$ and $\prod_{2}$ are said to be bisimilar with a precision $\varepsilon$, if there exists bisimulation relation, $\mathcal{R}_{\varepsilon}$ and for all $\left(\mathrm{s}_{1}, \mathrm{~s}_{2}\right) \in \mathcal{R}_{\varepsilon}$

\section{4}

American Institute of Aeronautics and Astronautics 


$$
\begin{gathered}
\mathrm{d}\left(\mathrm{O}_{1}\left(\mathrm{~s}_{1}\right), \mathrm{O}_{2}\left(\mathrm{~s}_{2}\right)\right) \leq \varepsilon \\
\left\{\forall \mathrm{x} \in \mathrm{X}, \forall \mathrm{s}_{1}^{\prime} \in \mathrm{S}_{1}\left(\mathrm{~s}_{1}, \mathrm{x}\right), \exists \mathrm{s}_{2}^{\prime} \in \mathrm{S}_{2}\left(\mathrm{~s}_{2}, \mathrm{x}\right) \mid\left(\mathrm{s}_{1}^{\prime}, \mathrm{s}_{2}^{\prime}\right) \in \mathcal{R}_{\varepsilon}\right\} \\
\left\{\forall \mathrm{x} \in \mathrm{X}, \forall \mathrm{s}_{2}^{\prime} \in \mathrm{S}_{2}\left(\mathrm{~s}_{2}, \mathrm{x}\right), \exists \mathrm{s}_{1}^{\prime} \in \mathrm{S}_{1}\left(\mathrm{~s}_{1}, \mathrm{x}\right) \mid\left(\mathrm{s}_{1}^{\prime}, \mathrm{s}_{2}^{\prime}\right) \in \mathcal{R}_{\varepsilon}\right\}
\end{gathered}
$$

Such bisimulation relations could be expressed as bisimulation function, $f_{B}$. The function $f_{B}: S_{1} \times S_{2} \rightarrow \mathbb{R}^{+}$is a bisimulation function between $T_{1}$ and $T_{2}$, if for all $\left(s_{1}, s_{2}\right) \in S_{1} \times S_{2}$

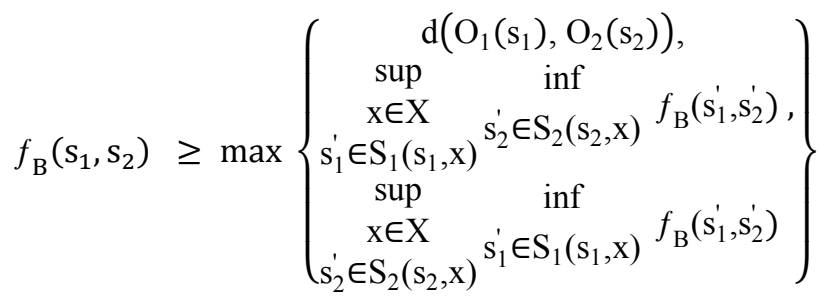

where the bisimulation function $f_{B}$ bounds the distance between the observations for a couple $\left(\mathrm{s}_{1}, \mathrm{~s}_{2}\right)$ by precision $\varepsilon \geq 0$ such that $f_{\mathrm{B}}\left(\mathrm{s}_{1}, \mathrm{~s}_{2}\right) \leq \varepsilon$ and non-increasing under operational dynamic conditions ${ }^{3}$.

\section{B. Consistent Behavioral Abstractions}

The abstraction precision in Eq.(10) between a concrete system and its abstraction is related to fidelity. This implies that abstraction is finding a surjection map and valid abstraction is finding the surjection map consistent with the SOU. To recall, Surjection ${ }^{\S}$ is an onto mapping where the codomain of a function is also its range. A function $\mathrm{f}: \mathrm{A} \rightarrow \mathrm{B}$ is a surjection means that every $\mathrm{b} \in \mathrm{B}$ is in the range of $\mathrm{f}$. Surjective functions here are used in mapping entities from high dimensional space to a low dimensional subspace.

Based on the propositions given in Ref.12 and from Eq.(7), the following proposition can be stated.

Proposition 1: For a given fidelity requirement, defined over some metric, $\delta_{F}$, the set of valid abstractions are given by

$$
\alpha_{\mathrm{SDU}} \mapsto \varepsilon_{\mathrm{SDU}} \mid \varepsilon_{\mathrm{SDU}} \leq \varepsilon_{\mathrm{SOU}}
$$

and the fidle abstractions are given by

$$
\alpha_{\mathrm{SDU}} \mapsto \varepsilon_{\mathrm{SDU}} \mid \varepsilon_{\mathrm{SDU}} \sim \varepsilon_{\mathrm{SOU}}
$$

From the definition of abstraction as surjection ${ }^{1,12}$, it follows that valid and/or fidle abstractions are member of the lattice, $\mathcal{L}_{\alpha_{i}}$.

It is well known from the behavioral systems theory that, the concept of states, similar to the one described in Eq.(8), is used to capture the necessary information about the evolution of a system. In other words, the problem stated above is how to partition state space of a dynamic system such that its abstracted semantics allows to conclude about its concrete semantics. Here the concrete semantics is assumed to represent real system.

In Ref.7, Julius et al presented systems behavior through state maps and states that relationship between bisimulation and lattice structure of the state maps. The abstractions or state map reductions are discussed through equivalence classes as position in the lattice. This study can be seen as continuation of this proposition and extends this bisimilarity preserving abstraction operation to the experimental frame formalism.

An important challenge of the system abstraction is the computation of bisimulation functions $f_{\mathrm{B}}\left(\mathrm{s}_{1}, \mathrm{~s}_{2}\right)$ between the system and its abstraction. This problem was addressed for constrained linear time invariant systems through the introduction of quadratic and truncated quadratic bisimulation functions ${ }^{3}$. It was remarked that the choice of surjection map to form an abstraction prior to constructing the approximate bisimulation function is

\footnotetext{
$\S$ Note that the word surjection and abstraction are used interchangeably in the context of behavioral compatibility
} 
heuristics based, provided, it respects the observation preserving and controlled invariant properties ${ }^{1}$. However, different such surjections are possible for the same level of abstraction leading to different precisions and if a certain precision is being desired by the user of the model, then it becomes important to relate this expected precision with this choice of surjection.

Thus, instead of choosing an admissible surjective map such that the precision of abstraction formalized as a semi definite optimization problem is minimal, the surjection map must be chosen such that the precision of abstraction is arbitrarily closer to the required precision. The existence of such an admissible surjection map gives the Necessary \& Sufficient (N\&S) abstraction consistent with the simulation objectives defined through precision, $\varepsilon_{S O U}$. From Eq.(11), the valid or necessary abstractions (N) i.e. the set of all admissiable surjections necessary for validity assessment is rewritten in terms of bisimulation function yielding precision $\varepsilon_{\mathrm{SDU}}$ as follows

$$
\alpha_{\mathrm{N}}:=\alpha_{\mathrm{SDU}} \mapsto\left\{\varepsilon_{\mathrm{SOU}}-f_{\mathrm{B}}\left(\mathrm{s}_{1}, \mathrm{~s}_{2}\right)\right\} \geq 0
$$

In conjunction with Eq.(12) \& (13), the sufficiency condition gives the abstraction with minimal granularity i.e. level of detail with respect to the objectives. Thus the the Necessary \& Sufficient (N\&S) is given by

$$
\alpha_{\mathrm{N \& S}}=\min \left(\alpha_{\mathrm{N}}\right)
$$

\section{Model Composition}

Models are usually composed hierarchically with interconnections between them and it is necessary to quantify the propagation of the effect of abstraction in composition. Due to the transitive nature of bisimulation functions, composition of bisimulation functions is possible, which in turn is amenable to standard engineering approach of hierarchical composition in system development.

Consider two systems which are approximately bisimilar and it follows that their respective abstractions are also bisimilar ${ }^{7}$. This entails that composition of resulting abstracted systems also will be bisimilar ${ }^{8}$ and thus providing bounds on the EF composed of different such systems. This morphism relation, similar to the one shown in Ref.7, can be seen from the following figure,

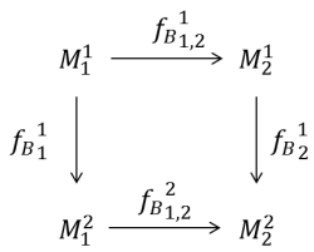

Figure 2: Composition of Bisimulations

It can be seen that the morphism relations are established between two models $\left(f_{B_{i, j}}^{n}\right)$ and also among the hierarchies of models $\left(f_{B_{i}}^{n}\right)$, where $\mathrm{i}, \mathrm{j}$ are models and $\mathrm{n}$ refers to abstraction operation. These compositional concepts will be further discussed in the context of applicability and derivability of the experimental frames in section V.A

\section{Consistent Abstractions of LTI Systems}

In this section, a class of dynamic systems, namely, Linear Time Invariant systems with (LTI) constraints on input and states is taken and abstractions consistent with simulation objectives are explained. Consider linear system with constraints on input, $x_{1,2} \in\left[x_{\min } x_{\max }\right]$ and state, $s_{1,2} \in\left[s_{\min } s_{\max }\right]$ described by state transition matrix $\mathrm{A}_{1,2}$ input matrix, $\mathrm{B}_{1,2}$ and output matrix $\mathrm{C}_{1,2}$ as

and its abstraction

$$
\begin{gathered}
\dot{\mathrm{s}}_{1}=\mathrm{A}_{1} \mathrm{~s}_{1}+\mathrm{B}_{1} \mathrm{x}_{1} \\
\mathrm{y}=\mathrm{C}_{1} \mathrm{~s}_{1}
\end{gathered}
$$

$$
\dot{\mathrm{s}}_{2}=\mathrm{A}_{2} \mathrm{~s}_{2}+\mathrm{B}_{2} \mathrm{x}_{2}
$$


where the abstraction is given by the linear surjective map, $H$, as $s_{1}=H s_{2}$

In such an abstraction operation, the map must be chosen such that it ensures propagation of desired properties such as stability, controllability and observability between the concrete system and its abstraction. Pappas et al in Ref.1 \& Schaft in Ref.4 proposed the extension of computer science notions of bisimilarity to dynamical systems and characterized it with controlled invariant concepts originally developed in the context of differential geometric control theory. Recalling the conditions for observation preserving partition ${ }^{1}$,

$$
\operatorname{Ker}(\mathrm{H}) \sqsubseteq \operatorname{Ker}\left(\mathrm{C}_{1}\right)
$$

The bisimulation conditions for linear continuous systems are given in the following equation

$$
\mathrm{A}_{1} \operatorname{Ker}(\mathrm{H}) \sqsubseteq \operatorname{Ker}(\mathrm{H})+\mathrm{R}\left(\mathrm{A}_{1}, \mathrm{~B}_{1}\right)
$$

where $\mathrm{R}\left(\mathrm{A}_{1}, \mathrm{~B}_{1}\right)$ gives reachablity.

It is remarked that in order for the partition to have the bisimulation property and thereby preserving transition, its null space must be a controlled invariant subspace. Controlled invariant space, introduced in the seminal papers of Ref.4, is a stability like notion where in the evolution of constrained dynamical system, constraint satisfaction can be guaranteed for all time iff the initial state is contained inside a control invariant set. However, as explained in section, this bisimilarity preserving surjections needs to be augmented with inclusion of objectives in the framework of designed fidelity.

\section{E. Finding Consistent Surjective Maps}

As explained in section III, Fidelity or Relative Fidelity refers to finding the necessary and sufficient abstraction with respect to requirements. Implicitly, such an abstraction must ensure semantics compatible with behavioral requirements $\left(\varepsilon_{\mathrm{SOU}}\right)$. This section explores the possibility of finding bisimilarity preserving fidle surjections using geometric systems theoretical concepts.

It has been shown that computing the coarsest bisimulation resulting in maximum complexity reduction corresponds to computing the maximal controlled or reachability invariant subspace inside the kernel of the observations map ${ }^{1}$. A coarsest partition of state space gives maximal controlled invariant set, $\mathrm{H}_{\max }$ and there exists a fix point algorithm to do it. The key question is the computation of a partition yielding desired precision instead of maximum complexity reduction by coarsest bisimulation. Since maximum controlled invariant set by its definition is the smallest possible set having all the invariant set, the computation of such map will result in an inclusion relation

$$
\operatorname{Ker}\left(\mathrm{H}_{\mathrm{SOU}}\right) \sqsubseteq \operatorname{Ker}\left(\mathrm{H}_{\max }\right)
$$

where $\mathrm{H}_{\mathrm{SOU}}$ is the reference user surjection which is mostly unknown and if known, fidelity becomes simply a problem of verification i.e. implementation.

The valid surjection maps are given by Eq.(13). However, quite often, fidelity needs to be optimal and the corresponding abstraction is called is Necessary \& Sufficient abstraction. The necessary conditions are given by Eq.(13) and the sufficiency conditions described in Eq.(14) then becomes,

$$
\operatorname{Ker}\left(\mathrm{H}_{\mathrm{SOU}}\right) \sim \operatorname{Ker}\left(\mathrm{H}_{\mathrm{SDU}}\right)
$$

Besides, a straight forward computation of $\mathrm{H}_{\mathrm{Sou}}$ may not be feasible since it is very difficult to obtain an abstraction without any knowledge of system functionality which in turn is different in different modes of operation. Instead, the approach should be finding criteria of maps which are valid with respect to requirements in addition to Eq.(19). However, there exists no fix point method to determine minimal controlled invariant $\operatorname{set}^{1}$ and similar argument can be applied to an invariant set existing in between the two extremum invariant sets.

The surjective criteria in Eq.(19) \& also limits the definition of SOU i.e. it gives a feasibility criteria. Considering the set of valid abstractions given by $H_{i=1 . . n}$ does not include $\varepsilon_{\mathrm{SOU}}$, it follows that,

$$
\mathrm{H}_{\mathrm{SOU}} \nsubseteq\left\{\mathrm{H}_{\mathrm{i}=1 . . \mathrm{n}}\right\}
$$


In other words, the abstraction is not feasible and the desired precision cannot be achieved.

It may be noted that, within an abstraction hierarchy, $i$, different abstractions, $\mathrm{j}=1 . .1$ corresponding to different invariant sets respecting bisimilarity conditions too can form a lattice similar to one described in section. But finding the coarsest bisimulation for each order $\mathrm{H}_{\mathrm{i}, \mathrm{j}+1}<\mathrm{H}_{\mathrm{i}, \mathrm{j}}$ might yield an idea about feasible precision such that if $\varepsilon_{\max } \sim \varepsilon_{\mathrm{f}}$, the partition is sufficient and if $\varepsilon_{\max }>\varepsilon_{\mathrm{f}}$, refinement is needed. The problem of finding surjections whose null space is controlled invariant subspace with respect to fidelity requirements needs to be explained in the M\&S standard of experimental frame formalism.

\section{Consistent Surjections of Experimental Frame}

As remarked in section III.A, Experimental frame describes scenarios under which a model is stimulated, observed and validated according to the requirements. It is worth noting that in general, either requirements or model is assumed right to validate its counterpart and in this paper, the focus is on how to abstract a model consistent with requirements. Thus many EFs can be formulated for the same SUT and the same EF can be applied to different SUTs. In this context of finding consistent surjections to build an EF, the two important EF concepts of applicability and derivability along with homomorphism becomes important ${ }^{9}$.

A morphism relation establishes correspondence between a concrete model and an abstract model and such a relation between two models is called homomorphism when the transition and output function has been preserved i.e. behavioural equivalence. A generic definition of abstraction operation is given by,

$$
\alpha_{\mathrm{j}}: \mathrm{EF}^{\mathrm{j}} \rightarrow \mathrm{EF}^{\mathrm{j}+1}
$$

where $\mathrm{j}=1 . . \mathrm{n}$ refers to the abstractions of a given $\mathrm{EF}$ as given in Eq.(6) \& (7).

Applicability and derivability are more structural concepts in that the former determines whether an EF can be applied to a model and the later determines the extent of such an application. Applicability and derivability defines compatibility criteria between a model and EF. Such EF concepts have industrial relevance too in terms of platform specific model reusability and building legacy models which are important factors in the complex system development. This compatibility is influenced by the abstraction level of EF components and the following sections discuss about it.

\section{A. Applicability \& Derivability of Abstractions}

In general according to the $\mathrm{V} \& \mathrm{~V}$ plan, different $\mathrm{EF}$ could be constructed representing the scenarios $\left(\mathrm{EF}_{\mathrm{i}} \mid \mathrm{i}=\left\{1 . . \mathrm{N}_{\mathrm{sc}}\right\}\right)$ for a given SUT. EF could be derived from a more general i.e. more capable EF and this operation of derivability is formally given by following definition

Definition: Derivability refers to ability to derive an EF from another EF.

$$
\beta_{\mathrm{k}}: \mathrm{EF}_{\mathrm{k}}^{\mathrm{j}} \rightarrow \mathrm{EF}_{\mathrm{k}+1}^{\mathrm{j}}
$$

where $\mathrm{j}=1 . . \mathrm{n}$ refers to the abstractions of $\mathrm{EF}$ as given in Eq. (20).

It must be noted that this set of derivable $\mathrm{EF}$ are included in the $\mathrm{EF}$ defined by $\mathrm{EF}_{i}$ as follows

$$
\mathrm{EF}_{\mathrm{k}}^{\mathrm{j}} \sqsubseteq \mathrm{EF}_{\mathrm{i}}^{\mathrm{j}}
$$

In other words, a scenario not present in $\mathrm{V} \& \mathrm{~V}$ plan could not be derived from the defined scenarios. The derivability is transitive due to the inclusion relation between them,

$$
\left(\mathrm{EF}_{\mathrm{k}+1}^{\mathrm{j}} \sqsubseteq \mathrm{EF}_{\mathrm{k}}^{\mathrm{j}}\right) \wedge\left(\mathrm{EF}_{\mathrm{k}+2}^{\mathrm{j}} \sqsubseteq \mathrm{EF}_{\mathrm{k}+1}^{\mathrm{j}}\right) \Rightarrow\left(\mathrm{EF}_{\mathrm{k}+2}^{\mathrm{j}} \sqsubseteq \mathrm{EF}_{\mathrm{k}}^{\mathrm{j}}\right)
$$

where $\mathrm{k} \in\{1 . . \mathrm{K}\}$ gives the limit of such operation. 
The relation between EF and SUT is given through applicability definition as follows

Definition: Applicability is an onto relationship between SUT and EF. Denoting the time segments by $\Omega_{\mathrm{L}} \subseteq(\mathrm{T}, \mathrm{L})$, where $\mathrm{L}=\{\mathrm{X}, \mathrm{Y}\}$ as defined in Eq.(3). Then applicability is a mapping such that the following conditions hold

$$
\gamma_{\mathrm{k}}: \mathrm{EF}_{\mathrm{i}}^{\mathrm{j}} \rightarrow \operatorname{SUT}_{\mathrm{m}} \mid\left\{\left(\Omega_{\mathrm{E}} \subseteq \Omega_{\mathrm{X}_{\mathrm{SUT}}}\right) \wedge\left(\Omega_{\mathrm{C}} \subseteq \Omega_{\mathrm{Y}_{\mathrm{SUT}}}\right) \wedge\left(\Omega_{\mathrm{LEF}} \vDash \emptyset_{\mathrm{EF}}\right)\right\}
$$

where $\mathrm{m}=\{1 . \mathrm{M}\}$ refers to the different SUTs according to the $\mathrm{V} \& \mathrm{~V}$ plan. In the applicability conditions given $\Omega_{\mathrm{E}}$ and $\Omega_{\mathrm{C}}$ are the experimental control segments to SUT and SUT observance segments respectively. The constraints applicable to states, outputs and inputs are given by $\emptyset_{\mathrm{EF}}$. Such compatibility conditions (syntactic \& semantic) are explained in the framework of trace inclusion in Ref.12.

EF is composed of different components and depending on abstraction level, different hierarchies of EF are possible for a particular scenario. Such a maximal applicable set of EF is given by

$$
\gamma_{1}^{\max }=\bigcup \mathrm{EF}_{\mathrm{k}=1 . . \mathrm{K}}^{\mathrm{j}=1 . \mathrm{n}}
$$

The question then becomes, similar to maximal controlled invariant set, how far a modeler can abstract the components such that the resulting EF is still applicable to SUT (whose validity is being assessed by simulation). In terms of surjection, what are the allowable surjections such that the resulting EF is still applicable to a SUT. In other words, what is the coarsest bisimulation possible between a model and its abstraction such that the abstracted EF is applicable to SUT. Implicit in this statement is the allowable bounds on the abstracted behavior i.e. abstraction precision. Applicability of an EF can thus be related by quantifying the abstraction.

Similar to Applicability, derivability can be described. Consider figure 3, where all these concepts are shown in a three dimensional lattice typology. For a particular EF, Interpreting derivability as an abstraction operation (horizontally in EF lattice), the set of all derivable EF i.e. different level of abstractions of the same EF components applicable to same set of SUT as the original EF gives the set of all valid abstractions of EF.

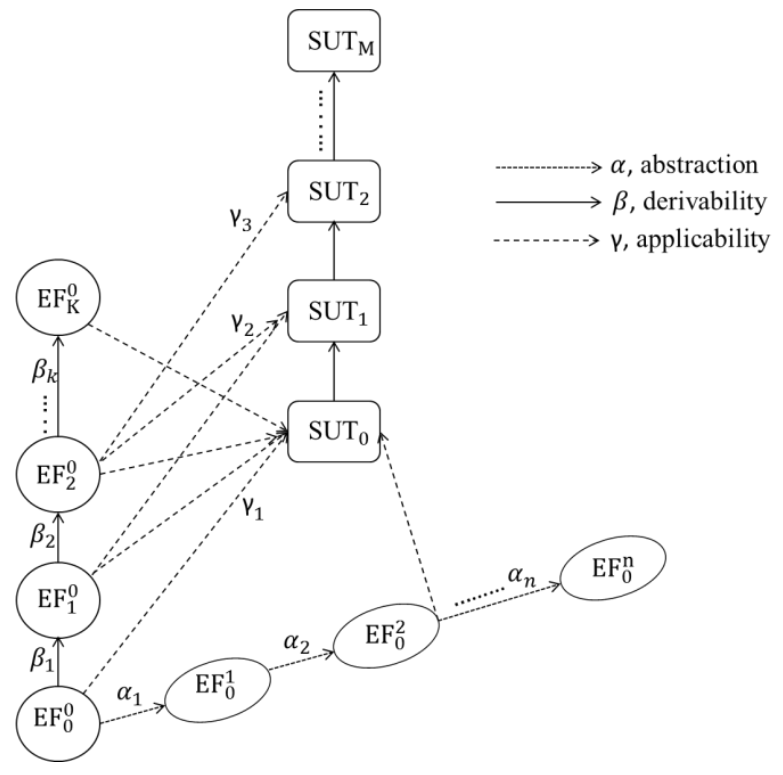

Figure 3: Applicability, Derivability \& Abstractions

Here all the derivable frames $\mathrm{EF}_{0 . \mathrm{K}}^{0}$ are applicable to a given $\mathrm{SUT}_{0}$ and the applicable abstractions (1..n) are the one which under abstraction still obeys this applicability. This applicability of abstractions can be seen for each derivable frame and abstractions must be chosen such that Eq. (7) is obeyed for the required scenario and given SUT. It can be noted that deriving from this EF upwards, the possible scenarios are fewer and the frames become 
more restrictive. In a sense, it is akin to abstraction in lattice i.e. the $\mathrm{EF}_{\mathrm{k}} \subseteq \mathrm{EF}_{\mathrm{k}-1}$ (scenario inclusion) and $\mathrm{SUT}_{\mathrm{m}-1} \subseteq$ $\mathrm{SUT}_{\mathrm{m}}$ (output inclusion).

Thus the problem explained in section IV.E can be rephrased as follows; the Fidelity could be posed as problem of finding controlled invariance of EF Model being abstracted (i.e. nullspace of surjection map) such that the resulting EF holds applicability property of the concrete EF. The relations between fidelity, abstractions are thus explained in EF formalism and concepts and a method based on formal notions needs to be developed to address these issues to build models with assured behavior on bounds and thereby ensuring fidelity.

\section{Conclusion \& Outlook}

This paper presents fidelity in EF formalism and gives a method for modeling the components through abstractions with the inclusion of end use objectives. A framework for synthesizing behavioral abstractions consistent with the simulation objectives in EF formalism is proposed and the problems were identified. The problem of finding surjections preserving bisimulation property as well as compliant with precision requirements was identified and few propositions were given. Further research is needed in better understanding this relation between abstractions and end use objectives, both in theoretical and industrial aspects.

In the current behavioral approach presented, in addition to the problems being studied, it must be noted that the language equivalence in bisimulation needs to be related in the EF context of transducer and acceptor. Since transducer and acceptor depend on observations of abstracted model, the equivalence criteria must be compatible such as EF cannot expect incompatible precision on outputs of an abstracted model. Also, the abstracted system can have different control input than original system ${ }^{10}$ and this need to be studied through the EF component abstraction such as abstractions of generator. The controllability and observability analogy of dynamic systems also have to explored in the context of experimental frame.

A unified fidelity framework needs to encompass capturing fidelity requirements and assessing the model fidelity through formal quantification of abstractions. In the long term objective of using simulation as a Means of Compliance for the system $\mathrm{V} \& \mathrm{~V}$, there has been ongoing works in capturing the fidelity requirements through IOU from the end user and consistently refining to build a use case through systems engineering tools such as SysML. In assessing fidelity, in addition to semantic compatibility described in this paper, methods such as ontologies are being studied to build rigorous simulation architectures for syntactic compatibility.

Formalization of fidelity problem as modeling abstraction will help improve the level of confidence in simulation results for the system $\mathrm{V} \& \mathrm{~V}$ and help better utilization of simulation resources by selecting the best available resource according to the test objectives. Identification of such a consistent and continuous way to improve simulation products will help improving product development life cycle quality while controlling the cost and mitigating risk. This Model-based systems engineering (MBSE) approach in developing simulation products is important in order to formally support the system life cycle development.

\section{References}

\footnotetext{
${ }^{1}$ Pappas, G.J, "Bisimilar linear systems”, Automatica, Vol 39, No 12, 2003, pp. 2035-2047.

${ }^{2}$ Pappas, G.J, Lafferriere, G and Sastry, S, "Hierarchically consistent control systems", IEEE Transactions on Automatic Control, Vol 45, No 6, 2000, pp. 1144-1160.

${ }^{3}$ Girard, A and Pappas, G.J, "Approximate bisimulations for constrained linear systems", Proceedings of Conference of and Decision and Control, 2005, pp 4700-4705.

${ }^{4}$ Basile G, Marro G, Controlled and Conditioned Invariants in Linear System Theory, Prentice Hall, 1992.

${ }^{5}$ Sarjoughian, H.S and Singh, R.K, "Building Simulation Modeling Environments Using Systems Theory and Software Architecture Principles", Advanced Simulation Technology Conference, April 2004, Washington DC., pp. 99-104.

${ }^{6}$ Van der Schaft, AJ., Equivalence of dynamical systems by bisimulation, Automatic Control, IEEE Transactions on, vol.49, no.12, pp.2160,2172, Dec. 2004.

7Julius, A.A. and Schaft van der, A.J., "State maps of general behaviors, their lattice structure and bisimulations", 16th International Symposium on Mathematical Theory of Networks and Systems, 5-9 July 2004, Leuven, Belgium.

${ }^{8}$ Girard A, A composition theorem for bisimulation functions, Technical Report, 2007.

${ }^{9}$ Zeigler, B.P, Praehofer, H and Tag, G.K, Theory of modeling and simulation, Academic Press, San Diego, California, USA, 2000.

${ }^{10}$ Girard, A and Pappas, G.J, “Approximation Metrics for Discrete and Continuous Systems”, IEEE Transactions on Automatic Control, Volume 52, Issue 5, 2007, pp 782-798.

${ }^{11}$ Ponnusamy, S, Albert, V, and Thebault, P, "Modeling \& Simulation framework for the inclusion of simulation objectives by abstraction", $4^{\text {th }}$ International Conference on Simulation and Modeling Methodologies, Technologies \& Applications, $\mathrm{N}^{\circ}$ 14453, 2014, pp. 385-394.
} 
"A Simulation Fidelity Assessment Framework", $4^{\text {th }}$ International Conference on Simulation and Modeling Methodologies, Technologies \& Applications, $\mathrm{N}^{\circ} 14452$, 2014, pp. 463-471.

${ }^{13}$ Haghverdi, E, Tabuada, P and Pappas, G.J, "Bisimulation relations for dynamical, control, and hybrid systems", Theoretical Computer Science, vol. 342, 2005, pp. 229-261.

${ }^{14}$ Tanner, H and Pappas, G. J, "Abstractions of constrained linear systems", Proceedings of the American Control Conference, Denver, CO, June 2003.

${ }^{15}$ Albert, V, Simulation validity assessment in the context of embedded system design, PhD Thesis, LAAS-CNRS, University of Toulouse, Unpublished, 2009.

${ }^{16}$ Roza, M, "Fidelity Requirements Specification: A Process Oriented View", Fall Simulation Interoperability Workshop, 1999.

${ }^{17}$ Foures, D, Albert and V, Nkesta, A, "Simulation validation using the compatibility between simulation model and experimental frame", Proceedings of the 2013 Summer Computer Simulation Conference, Vista, CA, Article 55, 7 pages, 2013. 